\title{
De una necesidad a posteriori
}

Genaro Aguirre Aguilar ${ }^{1}$

Quitó el mástil de la carlinga y enrolló la vela y la ató. Luego se echó el palo al hombro y empezó a subir. Fue entonces cuando se dio cuenta de la profundidad de su cansancio. Se paró un momento y miró hacia atrás...

Ernest Hemingway

Si naufragar es el único modo de conocer el océano, están permitidas todas las esperanzas...

Geneviève Brisac

El texto que aquí se presenta, es una suerte de capítulo crítico-reflexivo que forma parte de la tesis de Maestría en Comunicación Postal con nota atrás: antro y comunicación en los antros jarochos. La naturaleza de él, se desprende de un requerimiento institucional del programa de postgrado para reflexionar críticamente sobre lo hecho y no hecho a lo largo de la investigación. La posibilidad de proceder sobre ello, permitir voltear sobre el camino para hurgar en lo metodológico en tanto piedra angular en toda pesquisa de esta naturaleza. La forma de construcción es cual diario de viaje, donde las metáforas y un discurso "pretendidamente" narrativo trata de dimensionar lo académico. La intención de presentarlo para su publicación, no es otra que tratar de encontrar un eco en el campo del saber social construido desde la academia y el campo comunicativo, pues representa una opción para percibirnos y reconocernos en las ausencias y presencias; en las alegrías y los sinsabores que es la indagación social primeriza. Y aun cuando construir conocimiento es compartirlo, sin duda, este es un ejercicio muchas veces pendiente en las entregas de informes de investigación. He aquí otro tanto de los motivos, razón primera y última a la vez.

1. Universidad Cristóbal Colón. 


\section{Volver sobre los pasos ${ }^{2}$}

Con lo que a continuación se diga, es posible considerar concluida esta travesía.

Lo anterior se dice, porque cuando se inicia el navegar por alguna ruta que lleva al navegante a algún puerto, es posible saber los mares por donde cruzar, pero no los eventos que acompañaran ese andar fortuito: tormentas, marejadas, hastíos, renuncias, esperanzas, deseos, son acontecimientos que se van sumando a los días que terminan por integrar un diario de viaje, plagado de anécdotas, de recuerdos, de registros que son constancia de aquello que ahora pasará a formar parte de una memoria escrita. En ella, cartas, anotaciones, sueños, son los elementos que a la postre reviven para hacerse presentes cuando los recuerdo se cargan de tintes reflexivos, para reconocer desde el ayer recuperado, lo que fue correcto o incorrecto en esa travesía itinerante; siempre con una intención de fondo: hacerlos presentes para compartirlos e invitar a otros a cumplir con empresas similares, o para dar inicio a otras inquietudes que, como otras tantas anteriores, llevaron a andar por esos caminos ahora recuperados.

Casi así, lo que ahora se presenta es una suerte de "diario de viaje", en donde se pretende dar cuanta de los días y las noches que integraron ese navegar nocturno que como autor vivimos en este proceso de la investigación sobre las prácticas culturales que dan sentido a las formas comunicacionales y de interacción social forjada en seis antros del puerto de Veracruz. La necesidad de realizar esta búsqueda y presentación de lo hecho y no hecho, es sencilla: compartir una experiencia que contribuya a facilitar las rutas de viaje de otros que, igualmente, tengan la intención de trabajar con algo tan complejo como es la "realidad socialmente construida" e indagar sobre los mundos y representaciones del sujeto social, desde una perspectiva comunicativa; no sólo exponiendo cómo se construyó el Objeto de Estudio, sino también cómo se logró articular un marco epistémico y una metodología para la sistematización y análisis de la información que, con sus errores y asertos, condujo esta indagación, llevando a puerto una carga: la construcción de un conocimiento alrededor de la vida nocturna porteña.

2. Con la finalidad de encontrar un sentido y darle un fundamento a este apartado, tanto en lo académico (este es un trabajo de grado y como tal lo significativo del punto de vista del autor), como en lo epistémico (los elementos emic dimensionando la importancia de las formas lingüisticas del que puede echar mano el investigador inserto en un proceso de construcción de conocimientos, tal y como lo reconoce el paradigma interpretativo), en aquellos casos donde se juzgue pertinente el uso de la primera persona en plural así se hará. Después de todo, como ejercicio reflexivo es insostenible hoy buscar despersonalizar el lenguaje en aras de una objetividad, como se verá en el mismo cuerpo del apartado. 
A lo largo de este diario de viaje, seguramente se irán haciendo algunos altos en el camino para poder compartir mejor todo aquello que irrigó este ejercicio de búsqueda, síntesis, análisis, interpretación y reflexión sobre el abordaje realizado. Con ello, es justo reconocer que el esfuerzo no fue en vano, que los muchos problemas pudieron cargar energéticamente una actividad que principió como un sueño, para convertirse, en ocasiones, en pesadilla. Pero como quiera que sea, dio una experiencia que quedó allí, para bien y para mal y, sobre eso precisamente, queremos volver.

\section{De cuando se inicio la andanza}

Dicen que todo buen cuento inicia con "Había una vez..." Y en esta ocasión, la idea sería iniciar por ahí para contar las maneras en que fue abordada esta investigación, para lo cual tendríamos que remitir a esas primeras ideas que fueron acuñándose para convertirse en algo más concreto: el proyecto de investigación.

Era una de esas tardes tan comunes en el puerto cuando, ante el calor abrazante del medio día, una amiga sugirió ir a Los Portales. ¿Por qué no?, pensé, si después de todo tenía algún tiempo que no me daba la vuelta por esos lugares. Como en otras ocasiones, llegamos y el lugar escogido fue en la parte fuera del antro. Allí estábamos los dos, cuando comenzó a cruzar la idea que algo distinto (distintivo, incluso) estaba ocurriendo: mi amiga se quejaba que "de un tiempo acá", ese sitio se había puesto de moda; "de pronto, la clase bien de la ciudad", había ido ganando un espacio que ahora era moda visitar. Y sí, algo por ahí pasaba, pues de pronto el lugar común para la diversión y el solaz entretenimiento comenzó a ser Los Portales y, en términos generales, los antros de la ciudad.

A partir de allí, la idea se fue configurando para consolidarse en un anteproyecto que se presentó como requisito para ingresar a la Maestría en Comunicación, que de inicio se preocupaba por los "usos del espacio nocturno" en el puerto de Veracruz, pero que al primer contacto con los profesores de metodología del cuatrimestre de prerrequisitos, se redefinió. A partir de allí comenzó a ser de interés los antros. Como quiera que sea, un primer acercamiento con la oferta cultural para el consumo y la diversión, había llevado a recorrer las calles y rincones del puerto, de tal forma fue la exploración, que permitió tener un conocimiento afortunado de algunos procesos de socialización determinados por los habitantes de esta ciudad que, convertidos en agentes sociales, operaban y significaban al puerto de una manera distinta a la de ayer. 
De pronto, la aparición de una zona gourmet allí donde se conurbada Veracruz con Boca del Río, llevó a la reconfiguración de los hábitos alimenticios de la sociedad jarocha: los tacos y las carnes se convirtieron en parte del menú de una comunidad que de pronto comenzó a "tomar por asalto" la noche, donde degustar de este tipo de comidas junto al consumo significativo de licor, tejía una manera emergente de socialización y de práctica cultural significativa.

En esta dimensión, se dio inició la indagación: acudimos a diseccionar la ciudad en sitios considerados como más representativos de la vida nocturna. Cafés, salones de bailes, restaurantes, taquerías, discotecas, table dance, bares, principalmente. Con ello se estableció un primer acercamiento a los espacios y a las formas de vida e interacción en tales sitios. Igualmente, se llegó a la conclusión de establecer un cerco a la noche, pues la diversidad y la intensidad nocturna, terminaban por rebasar los tiempos y la energía que se desencadenaría en el proceso de investigación. Así las cosas, con una certidumbre que inquietaba, desde la "boca" de lo nocturno, surgió la idea de trabajar únicamente sobre los sitios que indistintamente se conocen como bares o restaurantes-bar o video bar, pero que para efectos de la investigación pasaron a convertirse en antros. El impulso de la gente y su constante presencia en sitios que la vox populi comenzó a llamar antros, supusieron una veta generosa para el abordaje de una ciudad dueña de una movilidad social que se configura en lo festivo y bullanguero.

Con algunos soportes teóricos que tenían que ver más con Antropología urbana, la Historia y la Sociología, se decidió por pensar la ciudad como un denso territorio que podía tener sus regiones como demarcaciones sociales para operar la vida nocturna y convertirla en un texto cultural para ser vista desde lo comunicativo. De tal suerte, se diseñó un Mapa Nocturno que convirtió a la urbe en algo simbólicamente más manejable: Zona Centro, de la Gran Barra Nocturna y de Marginales en el Centro, donde se seleccionaron 12 antros que de alguna u otra forma tenían una suerte de representación de lo que la ciudad ofertaba en términos de alternativas para la diversión a lo distintos grupos sociales, heterosexuales y homosexuales. Sin embargo, seguían siendo demasiados y, a sabiendas, que en una investigación de esta naturaleza siempre se termina por realizar cortes universales en ocasiones "dolorosos", se procedió a ello, quedando fuera los bares preferidos por uno de los sectores sociales más golpeados: los homosexuales. Se menciona esto, porque si bien es cierto en los antros que terminaron por ser considerados se buscó respondieran a aspectos generales y un tanto representativos de las situaciones y la lógica social desde la diversidad, también lo es reconocer que la ausencia de estos antros dejaba fuera a un sector que en los últimos años ha ido ganando espacios que antes eran mucho más difíciles de localizar en la ciudad, con todo y lo que Veracruz pueda ofrecer de liberal en un municipio que en los dos 
trienios que cerraron los 90 fue gobernado por los panistas; terminando por reproducir la discriminación de que son objetos socialmente hablando. Por lo anterior, se reconoce que antros como Yesterday, Deeper, Bue Le Carré, son apenas tres de estos lugares que exigen un análisis posterior para complementar esta postal de la vida nocturna porteña.

Después de esa selección, se dio inicio a una investigación que parecía afortunada por lo "novedosa". Se presentó el proyecto ante el Fondo Nacional para la Cultura y las Artes (FONCA) para solicitar un financiamiento que amortiguara los costos del trabajo de campo y la configuración teórica. En el tenor de las fortunas, se consiguió ese apoyo y con ello mayores facilidades, pero también más obligaciones y compromisos. Después tendría la claridad de lo que esto representaba. El trabajo profundo daba inicio.

\section{Echarse a andar la noche}

Allí estaban dadas las cosas para comenzar una navegación nocturna que llevara a esos rincones donde se sentía podían encontrarse ciertas formas de diversión que condensaban experiencias varias y complejas, las mismas que proponían un tejido de socialización donde se gestaba sentido y significado, que de una u otra forma mostraban ciertos rasgos de identificación característicos de la sociedad veracruzana: antros como espacios semantizados donde se configuran un entramado comunicativo intenso, articulado por disímiles formas de enunciación y significación.

Lo que ahora seguía, era fortalecer o definir mejor el objeto de estudio, ante la amenaza que representaba que el proyecto tuviera más que ver con la antropología y la sociología, que con la comunicación (aun cuando reconocemos el cruce disciplinario que sujeta la misma naturaleza de la investigación, descontando que muchos investigadores coinciden en señalar a la comunicación como el anclaje en la producción de conocimiento, cualquiera que este sea). Y esto en verdad no fue fácil, pues la mayoría de los textos hasta ese momento consultados, provenían precisamente de estas disciplinas. Sin embargo, conforme se accedió a otras lecturas, los tropiezos con algunos autores fue dando una perspectiva distinta sin renunciar a los aportes que, aquellas ciencias con muchos años de andar indagando sobre la ciudad, habían dejado un corpus teórico importante.

La ciudad dejaba de ser una mancha urbana y arquitectónicamente diseñada, ahora se convertía en un poderoso texto significado por la intervención de los ciudadanos convertidos en agentes sociales: en actores configurando una trama de significado y sentido que se podía estudiar desde la comunica- 
ción, siempre asistida por las otras ciencias que dotaban lecturas interesantes sobre la urbe.

Con esto, la definición hacia un objeto de estudio que tenía que ver con los usos y los formas comunicativas así como con mecanismos de interacción que se forjaban en los antros de la ciudad de Veracruz, esos espacios que se abren por la noches y se semantizan a partir de la incidencia de los asistentes que van convirtiéndolos es espacios territorializados; desde los cuales se teje una trama compleja pero textualizada que podía ser decodificada para dimensionar la estadía y la concurrencia a estos lugares, y con ello tratar de comprender las razones de la emergencia en las prácticas, los usos y consumos culturales de los antros, en los últimos años.

Así, por esos días, una serie de cuestionamientos se iban "agolpando" en la cabeza, ${ }^{3}$ llevaron a la determinación de nuestro problema de investigación: analizar las formas de articulación y reproducción de las prácticas culturales en los escenarios nocturnos, para comprender las estrategia de significación de los agentes sociales en la determinación de sus acciones, interacciones y procesos comunicativos durante sus estancias en los antros de la ciudad de Veracruz. De allí el planteamiento de una interrogante que entonces se consideró eje de la investigación: ¿Qué tipo de usos sociales y formas de consumo cultural se dan por las noches de fin de semana en 6 antros del puerto de Veracruz? Sin embargo, conforme se fue avanzando, esta interrogante sufrió una modificación gracias al trabajo de asesoría y tutoría que comenzamos a tener, complementado por el mismo proceso de descubrimiento, para dar pie a la pregunta central de la investigación, donde se potenciaba el trabajo hecho en campo: ¿Qué prácticas culturales dan sentido a las formas comunicacionales y de interacción social emergentes en seis ofertas de diversión y esparcimiento público durante las noches de fin de semana en la ciudad de Veracruz?

A la postre, esta pregunta se vería flanqueada por otras tantas que tenían que ver con los factores socioculturales, los sentidos y formas de socialización nocturna y el impulso urbanístico como facilitador de eso que comenzó a ser llamado mecanismo de interacción.

Con esas interrogantes a cuestas se fue "apuntando" la atención sobre los espacios abordados. Llevando como herramientas de trabajo inicial la etnografía; mientras que la entrevista a profundidad se pretendía como una herramienta que nos permitiría entrar en los universos de representación a través del discurso de los participantes nocturnos.

3. ¿Quiénes eran esos concurrentes? ¿Qué hacian allí? ¿De donde venían? ¿Cómo se relacionaban? ¿Qué tipos de usos le daban a determinados espacios? ¿Había una historia detrás de cada uno de los asistentes? ¿Cómo se podían definir?, fue el puñado de inquietudes base para la primera pregunta de investigación 
Diseñado el protocolo de etnografía y de entrevista, nos ocupamos de ir estableciendo los contactos pertinentes para ir descubriendo a los informantes claves. Sin embargo, las cosas se fueron complicando cuando el tiempo administrado se desbordó y amenazó el periodo mínimo para el nivel de observación requerido por la etnografía; junto a esto las entrevistas no iban saliendo. Apegados a los principios teóricos, procuramos establecer los contactos a través de las redes sociales, empleando como mediadores a los amigos o conocidos que decían "conocer" a una bailarina nudista, un cantinero, una chica "reventada". Y sí, los conocían.

Se hicieron los primeros contactos tal y como lo dictan los manuales, pero llegado el momento de la entrevista, se cancelaban o no llegaban. Estas, entre otras razones, fueron las que llevaron a replantear si era pertinente seguir o no en la búsqueda de informantes cualificados, en función que aquello iniciado como una entrevista a profundidad, eran charlas informales que nunca lograron ser lo pretendido.

Para entonces, el tiempo institucional, decía que teníamos que entregar resultados a la brevedad posible. Obvio, vinieron los primeros "tirones" con la calendarización académica, al reconocer que el tipo de investigación realizada no se podía medir de esa manera. El acuerdo fue replantear metodológicamente el tipo de procedimiento de origen, seleccionando de la etnografía una forma de registro procedimental llamada observación directa, descartando completamente la entrevista a profundidad, para atender con mayor énfasis las entrevistas in situ, que complementaron el registro hecho como investigadores.

\section{De noche y a oscuras, los miedos}

Si bien es cierto la consulta de textos iba solucionando algunos problemas, también lo es que iban articulando una trama más densa e intensa de análisis, que llevaba a sortear y ajustar sobre la marcha a propósito del proyecto ya encaminado. Aun cuando era una investigación cualitativa, se pedía una hipótesis que conllevaba una forma de validación académica, pero que hacía ruido cuando algún profesor decía que en este tipo de paradigma de investigación no era necesario, ni siquiera pertinente plantear hipótesis, una determinación metodológica enfatizada en algunas de las lecturas consultadas

Como quiera, la certeza era que las noches en el puerto de Veracruz se habían "abierto", y con ello una alteración de los patrones culturales nocturnos, devenidos con lo permisible de los 90; lo cual incidió en distintas esferas de la vida nocturna porteña: en los roles típicos de los agentes sociales, la configuración de los espacios y los tiempos de diversión, que habían llevado 
al establecimiento de nuevos sentido y usos de la ciudad. Esto, sin duda, debía provocar formas emergentes de interacción social, y tras esa búsqueda, anduvimos mucho tiempo.

La "certeza" de entender lo urbano como una cuña ideológica que atender para comprender y darle ruta a ello, llevó a rescatar lo "idílico imaginal" de la ciudad de Veracruz, de lo que se "dice" y piensa de ella, de lo festivo y relajado. Así, la ciudad porteña comenzó a ser vista desde el sentido común, trasladado a una abstracción teórica y conceptual que buscara darle forma académica, desde la comunicación, a este acercamiento, mitad gozoso y mitad en serio; aunque esto último fuera poco creíble. Permeando a esto, ya se vislumbraba la hermenéutica como marco metodológico en el análisis y la interpretación.

Como queda implícito, quizá la mayor claridad daba certeza, pero igual un puñado de incertidumbres. Y sí, comenzaron a ser muchas más de lo imaginado. De pronto las dudas iban en torno al rigor con que se estaba trabajando la observación directa, pues la necesidad de trasladar lo visto al texto sin amalgamar lenguaje, resultaba confuso en la medida de reconocer que la objetividad manifiesta en las acciones de los agentes sociales chocaba con la configuración discursiva del investigador, lo cual se reflejó en la forma de realizar el registro etnográfico. El reconocimiento de pertenencia a la comunidad discursiva que se estudiada, fue el primer ejercicio de reflexión profundo hecho, y que llevó a la consulta de autores para establecer claridad en el entendimiento de este fenómeno y, por el otro, para encontrar estrategias de solución a ello con la intención de ser más precisos, con apoyo de autores como Spradley (1980), Geertz (s/f), Thompson (1996), Poblete (1999), entre otros. Con ello, se trabajó con más rigor y precisión, y así se "amarraron" formas para diseccionar las lógicas sociales de los espacios investigados sin alterarlas. Más tarde encontraríamos a autores como Renato Ortiz (1999) y Sergio Poblete (1999), para quienes hablar de "objetividad" a la hora de registrar acciones sociales, es un problema de legitimación académica de la investigación cualitativa, que busca hacerse de ellas frente a la otra perspectiva teórica; donde incluso, aflora una "falta" de reconocimiento de que somos sujetos configurados discursiva y contextualmente.

Por otro lado, la parte de las entrevistas, seguían sin funcionar. Contactos abortados que no tenían otra forma de explicarse que no fuera la falta de pericia por parte del investigador para entablar un diálogo. Ya entonces, la oportunidad de ingresar al proyecto de investigación en red, representó la oportunidad de tomar talleres de capacitación, que provocó el reconocimiento que para entrevistar a los personajes, podía utilizar un elemento complementario de la etnografía como es la entrevista etnográfica en sus tres niveles: in situ, etnográfica y relato etnográfico. Sólo había un problema, la falta de 
tiempo, algo que se hizo saber en uno de los seminarios convocados en la maestría.

Para entonces, otros problemas comenzaban a hacerse presentes. De los seis lugares seleccionados: La Tasca Colonial, El Rincón de la Trova, Ocean, Chévere Cocó, Lencerías y Kokai, uno había cambiado de giro sin que se cerrara el primer ciclo de acercamiento a ese lugar (se pensaban fueran mínimo 2 y máximo 4 visitas): Lencerías, pasaba a convertirse en un antro donde se presentaba un espectáculo table dance. Y como lluvia, se dejaron caer otras circunstancias fortuitas y no deseadas: en la discoteca Ocean, después de tres exploraciones, ya no tuvimos acceso por lo que pensamos era un acto de discriminación, situación hecha público en un par de periódicos locales a los que dirigimos sendas cartas de protesta. Para esto, antes en Kokai, se había descubierto la presencia de alguien que no necesariamente iba a divertirse. Invitados a pasar al área de administración, intercambiamos puntos de vista con el responsable, a quien se le dijo la naturaleza del trabajo. Aún entendiendo esto, nos invitó a considerar la privacía de muchas de las chicas, a quienes les incomodaba este tipo de trabajo. Como quiera, nos propuso que acudiéramos a solicitar autorización con los "jefes".

Entrevistas con el administrador y con el representante de los artistas en la ciudad, fueron una experiencia diferente: con el administrador no pudimos hablar, mientras que el representante de la Asociación Nacional de Actores (ANDA) delegación Veracruz, dijo que no había problemas.

Llegados aquí, se siente la pertinencia de hacer un alto en el camino para analizar los logros y fracasos que acompañaron esta parte de la investigación; donde se buscará cruzar lo dicho por la teoría y las "travesuras" que la realidad prepara a quien se mete en los complejos caminos del estudio de lo social.

\section{Navegar sin rumbo}

Sin lugar a dudas, uno de los aspectos significativos que creo los participantes de este programa de postgrado vivimos, fue consolidar de un cuerpo académico propio que otorgara tutorías metodológicas a nuestros proyectos de investigación. ${ }^{4}$

Se dice esto y pensamos en el caso nuestro, pues la ausencia de un director de tesis y tutor metodológico durante los cuatro primeros cuatrimestres, fue clave. Andar los caminos de la investigación suponiendo, haciendo intuicio-

4. Es claro que lo dicho aquí, tiene que ver con una percepción personal, y como tal asumimos lo dicho. 
nes, dando tumbos de aquí para allá, fue un proceso desgastante: energética e intelectualmente. Desde la falta de recomendaciones sobre el proyecto y los protocolos entregados, a la renuencia absurda de nosotros para acercarnos a solicitar orientación con la coordinación del programa, terminaron por afectar el mismo proceso de investigación: el tiempo para fortalecer el trabajo de campo que exigía el tipo de investigación, ${ }^{5}$ resultó ser una de las "variables extrañas" que amenazó el trabajo; sin embargo, las mismas características de la perspectiva de abordaje (la flexibilidad metodológica), facilitaron el replanteamiento en el programa de trabajo.

Así, el replanteamiento en la estrategia metodológica al presentarse problemas del orden operativo, terminó por ser un facilitador frente a esta situación: de la etnografía planteada al inicio, pasábamos a la observación directa, mientras que en el caso de la entrevista a profundidad, pasábamos a la obtención del discurso de los agentes sociales a partir de una serie de entrevistas in situ. Esto llevó a la adecuación del protocolo inicial, lo que generó la búsqueda y lectura de material que enriqueció este nuevo estadio metodológico.

En este mismo sentido, si bien es cierto - como ya se dijo- al principio la retroalimentación falló, conforme hubo la cercanía con nuestra directora de tesis, pudieron revisarse los documentos para corregir los problemas (el tan nombrado rigor metodológico que andaba a la deriva, comenzaba a enfatizarse gracias a esta nueva condición de trabajo). ${ }^{6}$ Como parte del trabajo de asesoramiento, se replantearon el marco teórico, el objeto de estudio, las preguntas de investigación, enfatizando en la perspectiva comunicativa, ante la amenaza de producir una tesis con otra orientación. Este aspecto, fue realmente importante.

En el caso del marco teórico (que respondía al índice que se había presentado en el proyecto original), se tuvo que modificar, suprimiendo un número de temas que para su configuración teórica habían exigido la lectura de textos y su correspondiente comprensión, pero que en su incorporación se presentaban "desarticulados" entre sí y con el objeto de estudio. Aun cuando esto llevó a otra búsqueda y a inversión de un tiempo que se podía haber empleado para "otras" cosas, los resultados, estamos seguros, fueron mejores.

5. Sumado a que el cuerpo teórico no tenía articulación y habría que suprimir una buena parte de él, terminó por requerir una inversión de un tiempo con el que para entonces no contábamos.

6. Sin embargo, asumiendo una posición ante esto, es responsable y honesto reconocer que mucho se dio por la falta de confianza mutua. Así, si no tuvimos director o asesor durante los primeros cuatro cuatrimestres, supusimos "nosotros podíamos" sacar adelante el proyecto sin la guía de un especialista. Y así, mientras el tiempo seguía adelante, las cosas se complicaron. El barco salió adelante, cuando llegó la asistencia tutorial. 
Por otro lado, el programa académico exigía cumplir con los tiempos. Teníamos que estar entregando borradores y avances, que en muchos casos no fueron ni lo uno ni lo otro. En nuestro caso, se optó por dejar que los tiempos corrieran, ya con una decisión tomada: no se podía entregar algo que no teníamos idea cómo se debía entregar, pues el borrador implicaba conclusión de etapas y, por lo menos, nosotros no lo teníamos y no considerábamos responsable entregar trabajos a "medias".?

Junto a esto, algo quedaba claro de inicio: el proceso había sido complejo por la misma condición en que se encuentra quien por primera vez realiza un trabajo como este; no sólo porque hay "lagunas" formativas de carácter curricular y profesional que inciden en la concepción que se tiene de la comunicación en todas sus dimensiones, ${ }^{8}$ por un lado y, por el otro, las "debilidades" metodológicas que se presentan. Porque una cosa es cierta: se puede tener acceso a una extensa bibliografía, pero pasar ese cuerpo teórico al trabajo de campo, en muchas ocasiones se convierte en un "salto mortal" sin "malla" protectora, ${ }^{9}$ que termina por provocar un accidente bastante doloroso.

Ante todo esto, el fortalecimiento en la interacción con aquellos especialistas y responsables de la asistencia metodológica, hubiera permitido el conocimiento e involucramiento mejor con el trabajo, para con ello generar encuentros significativos al unir sus conocimientos teóricos con la perspectiva "real" y sistemática que puede ir adquiriendo con el trabajo de campo el responsable de una investigación, quien se va convirtiendo en "especialista" temático en la "talacha" diaria de campo, pero que le falta esa "talla" propia

7. Entre las etapas que faltaban, estaba el capítulo fuerte, donde se tenía que analizar, interpretar para convertir en texto todo el trabajo teórico y empírico, que por otro lado, ni siquiera sabíamos por dónde y cómo configurarlo, pues cada uno de esos momentos, si bien parecen iguales, tienen sustanciales diferencias. $Y$ eso, en muchos textos, no lo encontramos. Igualmente ocurría con el capítulo crítico, donde se tenía que proceder al esclarecimiento de todo el proceso de investigación, en el cual se debía exponer la experiencia, los errores cometidos, las decisiones metodológicas, las formas en que se construyó el conocimiento adquirido y en qué había cambiado la percepción y la parte humana de nosotros. En todo ello, había demasiadas incertidumbres sobre lo que se podía decir, reconocer y asumir como responsables de un trabajo de esta naturaleza.

8. Se hace referencia directa a este campo disciplinario y al programa de maestría en el que se ubica esta investigación, pero quizá no resulte extraña y ajena a otros. Incluso, un alto porcentaje de las licenciaturas en comunicación, parecen apostar más por la formación técnica, si tomamos en cuenta las currículas y las "inquietudes" de los egresados, ambos por encima de la perspectiva teórica y de investigación propia de los estudios superiores. La cientificidad sometida a la técnica, pareciera el síntoma en estos tiempos.

9. Para ello, en los niveles institucionales se deben reconocer como algo propio del proceso formativo estas ausencias, pues esta certidumbre -que vaya más allá de las palabraspermitiría diseñar un programa fuerte de asistencia metodológica que fortalezca y dé "claridad" en quienes por primera y quizá única ocasión, se verán insertos en dinámicas de trabajo como estas. Con todo ello, un montón de interrogantes podrían ser respondidas. 
del rigor académico. La necesidad de encaminar la asistencia metodológica por estos "rumbos", sin duda hubiese facilitado la administración de energías de ambas partes, además de tener como producto terminal la calidad en los resultados posibles.$^{10}$ Por otro lado, entendemos y reconocemos (incluso se dijo desde el principio), que la metodología cualitativa es afortunada, en virtud de lo flexible de su diseño; sin embargo, sostenemos que la experiencia de formación que representa el trabajo de investigación, hubiera sido distinta, para evitar decir mejor.

Como quiera que sea, esto fue el inicio, pagamos la novatez; que a todos les pasa esto; pero en descarga de ello que va siendo del lugar común, creo es necesario poner "candados" a partir del compromiso compartido, donde el ideal de la construcción de conocimientos, de la configuración de redes de sentido, realmente opere en todas direcciones. Y que no se quede únicamente en "buenas intenciones" o en discursos que, se suponen, en lo académico deberían ser evitados. Atrás han quedado rutas, itinerarios, esbozos de algunas intenciones que ya no se cumplieron, pero de las que se obtuvo un aprendizaje; ahora queda hablar de las cosas que dejaron huella: cómo se construyó el conocimiento, desde la problematización, lo metodológico, el análisis y los métodos que estratégicamente se conjugaron para sacar adelante el trabajo. Sobre el conocimiento personal y las aportaciones que este trabajo hace a la sociedad.

\section{De lo recogido en ese andar}

$\mathrm{Y}$ como todo andar, cada viaje tiene su final.

De todo esto, la experiencia más grande que queda, es haber descubierto que era posible estar cerca de un proceso de investigación, donde uno era parte de él, en dos sentidos: como sujeto cognoscente y reconocido. Y junto a nosotros, otros más también preocupados por ser partícipes de esta experiencia.

Descubrir otros mundos a través de los ojos y los sentidos, la teoría y la reflexión, seguro nos ha cambiado la vida. Apostar por un tema sobre el que se quería encontrar fundamentos epistémicos y teóricos, no fue tarea sencilla. "Subir a la montaña" para ver el mundo de otra manera, es un recorrido en el que se termina por creer, cuando se ven productos: lecturas, discusiones,

10. Algo que por ejemplo nunca se supo, es la razón del porqué un alumno como responsable de un proyecto de postgrado, no tiene la posibilidad de escoger a su asesor tal y como ocurre en casi todos los programas de maestría. Quizá tenga que ver con una cuestión institucional, sin embargo faltó ese diálogo para tenerlo en conocimiento. Esa es una situación que bien podría cambiar para generar acciones facilitadoras encaminadas al mejor cumplimiento de los proyectos. 
amigos, experiencias compartidas, el reconocimiento de los "otros"; de la ciudad, de la noche, del ejercicio sistemático de pensar y pensarse para configurar discurso reflexivo, es algo que terminamos por agradecer.

Cómo dejar de lado las noches y los días de andar a la deriva en la lectura, de andar a salto de mata tras las tesis que sentíamos eran el rumbo para ir definiendo mejor el objeto de estudio; para tratar de demostrar que el antro y sus maneras de nombrarlo a través de las acciones y enunciaciones sociales, eran un asunto de interés personal pero igual compartido desde una perspectiva humanista y académica.

Por otro lado, la tarea de configuración teórica-conceptual, llevó por un mar de información -a la postre ponderado por la amenaza de no obedecer a la articulación propia del proyecto de investigación-, que sin duda trajo hallazgos teóricos que "reventaron" los niveles de lectura vividos antes. Aprender a hacer a un lado datos, informes, que al inicio se consideran como necesarios, es un acto de (in)certidumbre metodológica que sólo se obtiene ante la ocasión de hacer frente a este tipo de decisiones, que exigen un re-visión, que devienen en una experiencia cognitiva rica y compleja. Eso, no se puede olvidar.

Sumado a esto, saber que el conocimiento se construye socialmente y en el intercambio de puntos de vista, es un acto generador de oportunidades al que la investigación abre la puerta para hacernos cada día mejores. Junto a esto, el reconocimiento del proceso de aprendizaje y la recuperación de los estados de asombro, es una dinámica que no encuentra mejor punto de confluencias, que el estar frente a eventos que son registrados voluntaria y racionalmente, en la inmersión de una cotidianidad que se revela compleja, caótica... expectante.

Sí, decimos esto pensando en el trabajo realizado, el mismo que obligó ir articulando discurso a lo largo de los días, reflexiones acumuladas que iban llegando a "puerto" conforme íbamos creciendo en la pesquisa. Cómo no recordar cuando volvimos al proyecto a sugerencia de nuestra asesora, para darnos cuenta que el camino se "mostraba" difícil y habría que atender mejor. Cómo no traer aquí aquellos días cuando la realidad -la misma que se revelaba tozuda y decía que no se dejaría "coger" tan fácilmente- nos sorprendía y obligaba a dar un giro en el timón. Así, la vuelta a las interrogantes-luz, provocaba cambios sustanciales, pues posteriormente eran replanteadas ante las mismas dinámicas de descubrimiento y el impulso generado por la reconfiguración del corpus teórico que llevó por otros derroteros y categorías de aproximación a lo social.

De tal suerte, para hablar de la construcción de conocimientos, tendríamos que apelar a las interrogantes, pues es sabido que el mínimo indispensable para iniciar esto, es la incertidumbre e "interrogancia" frente a los eventos que se nos revelan. Sin embargo, para llegar a ellas, teníamos que plantearnos 
un problema de investigación, algo que para entonces no teníamos claro y que se revelaba complejo, si tomamos en cuenta que, en la formación que podamos tener, no establecemos distingos entre un problema práctico y el de investigación; ${ }^{11}$ y lo que es más, muchos manuales de metodología de investigación, no establecen con asertividad esto.

Así, tratar de establecer una confluencia entre los conceptos básicos, el objeto de investigación, la perspectiva a priori que teníamos sobre el fenómeno de interés, fue un ejercicio intenso en los niveles conceptual, semántico y cognitivo. Pues fue verter sobre el papel un ejercicio sistemático de síntesis de las lecturas incorporadas, la re-visión del objeto de estudio para poder construir teóricamente un problema de investigación. Algo que se dimensionó y construyó cuando se exigió y orientó, a partir del trabajo de asesoría, donde el referente sistematizado fue el modelo heurístico; esa trama relacional que hizo visible lo conceptual (desde lo tangible e intangible) y estructural (sociedad, representación social y comunicación y cultura) en sus múltiples dimensiones y niveles.

Desde allí, la pregunta: ¿Qué prácticas culturales dan sentido a las formas comunicacionales y de interacción social emergentes en seis ofertas de diversión y esparcimiento público durante las noches de fin de semana en la ciudad de Veracruz?, se definía mejor, lo que permitió dar la base para generar otras tantas, tales como: ¿Qué factores socioculturales han generado una redefinición de los roles típicos de los personajes nocturnos en el puerto de Veracruz?, ¿Cuáles son los nuevos sentidos y formas de socialización nocturna que se esbozan en la ciudad de Veracruz, como formas emergentes de interacción?, ¿De qué manera los "personajes" nocturnos comparten, negocian, establecen, delimitan, construyen sus identidades y definen el territorio en un antro? Con estas interrogantes, se potenció mejor el trabajo, y ante ello, los resultados fueron más enriquecedores para la producción de conocimientos que buscábamos.

La generación de estas nuevas interrogantes a propósito de la redefinición y consolidación de lo andado, por una parte, y el reconocimiento de la ingenuidad de las anteriores ante los problemas ya expuestos, por el otro, lejos de causarnos una duda académica, provocó un fortalecimiento que llevó a un "punto" de complejidad que emergió en el proceso mismo de indagación. Por primera vez en muchos meses, había una satisfacción en lo que se estaba

11. Un asunto que pareciera fácil por de términos en apariencia, sin embargo, en términos reales tiene que ver con lo conceptual y operativo, en la medida de ser un ejercicio que debe cumplir con las características propias de la comunidad científica y el proceder legitimado en el uso de un lenguaje, que valide la tesis como trabajo académico. Algo sobre lo que esperamos volver un poco más adelante. 
haciendo. Por primera vez, se pensaba estábamos cerca de un trabajo recepcional de postgrado.

\section{Los métodos y sus estrategias: cartas esféricas del navegante}

Algo que queremos atender por lo significativo, es la parte de la sustentación de todo lo hecho en este trabajo, donde dos mundos se revelan próximos, pero igual distantes; similares pero diferentes en algo: su punto de partida y el puerto de llegada. Nos referimos a lo científico y a lo académico: al proceder fundamentado para llegar a la obtención de un producto terminal como es la tesis de grado, donde el autor tiene que buscar legitimar el "fondo" de su producción con una propuesta fuerte de sustentación teórica, pero que en la "forma" tratará de establecer su canon de legitimidad desde su propio ámbito de validación.

¿Por qué dedicar un apartado en el capítulo de valoración a estas dos formas de producción de conocimiento? Porque lo que se va a decir tiene que ver precisa y fundamentalmente con ello. Así, en esta parte donde hablaremos de los métodos de trabajo empleados, las estrategias de abordaje y análisis para la producción de saberes, es necesario dejar asentadas algunas inquietudes recogidas en nuestro andar y que obligaron a tomar una posición crítica, reflexiva y discrecional sobre lo científico y aquello que es académico.

Partiremos de un hecho: en un trabajo de investigación, los clásicos piden que haya en sustento teórico (conceptual, referencial, metodológico, etc.) para poder reconocer en ese proyecto algo que se valide desde el universo científico. Cosa similar ocurre con un trabajo de tesis. En ambos, hay un procedimiento de legitimación que tiene que ver con el rigor y el uso correcto de las formas discursivas. Así, estos mundos "diferentes" se acercan en ello, pero se distancian en las formas de entregar sus productos.

Para este trabajo de investigación, la naturaleza y la forma en que se construyó el objeto de estudio, exigió el conocimiento del llamado método cualitativo, que requirió una ubicación epistemológica que nos llevó al conocimiento de otras posibilidades de abordaje, desde la necesidad de establecer racionalmente las diferencias entre métodos, metodologías y algunas de sus técnicas. La búsqueda de esto, puso en claro que cada uno de ellos, tiene su forma de validación, no sólo por el procedimiento sino también por el lenguaje empleado, que se remite a sus propias raíces epistémicas y relacionales con los fenómenos estudiados.

Así, desde la perspectiva interpretativa, iniciamos el trabajo para indagar sobre los antros, los ciudadanos y sus formas de interacción social. Paralelo al 
trabajo de campo, la incorporación de lecturas que tenían que ver con el interaccionismo, la fenomenología, la etnometodología, la hermenéutica; sin llegar a tener la proyección que acabaríamos con la necesidad metodológica de considerar cuatro métodos, a partir de la propuesta de un mismo autor: el crítico-racional, el cualitativo, el histórico y el comparativo. Reconocemos que nos preocupaba no tener la información suficiente, por lo menos de dos métodos: el crítico-racional y el comparativo. Pero era una necesidad académica sustentar el trabajo en estas propuestas. Y no lo cuestionábamos, pero sí llamaba la atención que sólo tuviera que ver con el método y en menor medida con lo metodológico, ¿y lo epistemológico, de dónde lo podíamos obtener?

He aquí un problema que encontramos: el oficio del investigador primerizo se hace difícil y complicado, cuando no se reconocen las dimensiones del conocimiento, y sólo se remite a lo operativo. La estructuración cognitiva no hace "clic", si sólo se remite la búsqueda de información práctica y no a las bases epistemológicas de esos procederes. Sumado a esto, la variedad de formas de nombrar las cosas por parte de cada uno de los autores con los que se puede uno tropezar, y que suponen una autoridad en la materia, pero que pocas veces se reconocen como miembros de una escuela, corriente y por lo tanto de un back ground cuyo soporte es lo ideológico, pocas veces reconocido. Después de todo, la generación de los tipos de conocimientos, desde aquí se sostiene, se legitima, se determina. $\mathrm{Y}$ eso, como primerizos, a veces no lo ponemos en perspectiva.

En el caso de nuestro trabajo, sabemos encontramos esa legitimación, sabemos se estableció la lógica de abordaje, análisis, interpretación y textualización a partir de un ejercicio de discernimiento, que condujo a tener claridad sobre la diferencia en cada uno de esos estadios; por lo cual, cada uno nos exigió su validación teórica. Esto fue fundamental en el proceso de aprendizaje vivido.

Pero aquí caemos en algo que resulta inconsistente. Mientras desde la academia se buscaba un soporte teórico en estas corrientes del pensamiento universal científico, por el otro, no se reconocía como viable el empleo de un lenguaje reconocido como válido por la perspectiva de abordaje que condujo al estudio del objeto de investigación. El hablar en tercera persona buscando ser objetivo con ello, consideramos es tratar de asirnos a un reducto insostenible, ya muy debatido en el terreno científico contemporáneo. El nivel de complejidad a la que llevan los nuevos paradigmas cuando se unen a lo cultural y científico, va más allá de este acto reduccionista y ceguera intelectual del mundo académico; a lo que se suma, la coincidencia de saberes de distinta índole (desde lo biológico a lo estético), pero que parece lo académico está negado para reconocer esto; aun cuando un trabajo de tesis de grado -como es este caso-, debe exigir una toma de posturas del autor, sustentadas 
en el ejercicio riguroso de su proceder y la fundamentación de sus argumentos. Pero lo que por derecho se tiene, se niega, aun cuando buen tramo del camino, se busque validar lo dicho, con otros dichos que la ciencia (histórica y actualmente) viene entregando en la producción de conocimientos. En este mismo tenor, se procedió en su momento para el análisis, interpretación y textualización de todo lo obtenido. Un poco más adelante volveremos sobre esto.

A partir de lo anterior, algo que buscó caracterizar al trabajo fue su estilo en el discurso, sin duda más narrativo y literario, ${ }^{12}$ pero desde el cual buscábamos evidenciar un trabajo analítico, reflexivo, producto de lo trabajado en campo y en la revisión teórica; donde las categorías de análisis iban tomando anclaje en el encuentro con los agentes y sus acciones, para terminar trasladándose al cuerpo enunciativo que iba estructurando en lo escrito, un texto que consideramos fundamentalmente académico

Así, hasta dar con una suerte de intentos conclusivos que buscaron (des)articular el corpus informativo de cada uno de los sitios, para finalmente integrar un escrito que holísticamente nos diera esa postal con nota atrás de la ciudad de Veracruz, más allá del imaginal universo al que remite este nombre cuando en algunos espacios lo referimos. Como quiera, sabemos que apenas eran guiños con un intento de perspectiva teórica.

\section{Se hizo camino al andar}

Ya sin pretender superar lo no hecho, ${ }^{13}$ de común acuerdo con nuestra asesora y directora de tesis, terminamos por reconocer que nada era más conveniente que iniciar el proceso de análisis sobre el material que se tenía. Para esto, mientras nos sugería un análisis argumentativo, nosotros pensábamos, dudábamos en virtud de carecer de discurso; por otro lado, apelando a la "red"14 habíamos establecido contacto con algunos miembros y ellos nos

12. Este aspecto resulta fundamental, pues la confluencia de un discurso más cercano a lo literario y propio de la academia cra la pertinencia para un estudio sobre lo cotidiano, aun cuando al principio se nos sugirió cambiar el estilo, una manera escritural que se fue forjando a lo largo quince años, que tenía que quedar aquí presente. Sentíamos que el gozo vivido en la investigación era más vivo si lo facilitábamos a través de la escritura; después de todo, el "rigor" tiene que ver con "otras" cosas que ya quedaran expuesta.

13. La referencia directa es al tiempo de observación para desarrollar un nivel de profundidad de acuerdo a la exigencia metodológica que nos planteamos al inicio.

14. Red de Investigación y Comunicación Compleja (RICC), agrupación que aglutina a un número de investigadores nacionales y extranjeros, que son la base académica en el programa de posgrado en Comunicación de la U.V., con una visión distinta de lo que es y debe ser la investigación 
invitaban para reconsiderar este tipo de análisis, en virtud de no estar trabajando sobre textos discursivos. Después de comentar esto con nuestra asesora, llegamos al acuerdo de hacer conjuntamente una propuesta de análisis, no sin recomendar el sustento teórico que nos diera solidez. Afortunadamente encontramos un par de reportes donde el tipo de observación y la propuesta de análisis se parecía a lo que pensábamos se podía hacer. Si al principio resultaba "pequeño" y sencillo, llegamos a fundamentar mejor la propuesta de análisis, interpretación y textualización de la información.

Se partió del hecho que, ante la falta de tiempo y registro en sus distintos niveles de profundidad, lo pertinente era buscar obtener un producto descriptivo como un primer nivel de acercamiento al objeto de estudio. Ante la certeza de lo flexible de la metodología, no veíamos por qué no realizar un replanteamiento en la configuración de la técnica de análisis. Así que la tarea fue proponer una mecánica de abordaje en su nivel comprensivo.

En este sentido, cabe señalar que, si bien es cierto la tecnología había venido a contribuir enormemente en nuestro trabajo, también lo es que en la parte de vaciado de la información nos negábamos a emplear un programa sobre el que no teníamos dominio. El probar una y otra vez el Excel, provocaba una angustia que terminamos por superar cuando decidimos hacerlo como se hacía "antes", a mano; vamos, artesanalmente. La decisión fue una estrategia acertada. Así lo hicimos ver a nuestra asesora, a quien le preocupaba -justificadamente-, que la información obtenida con esa técnica de sistematización, se presentara como "sacada de la manga". Por supuesto aseguramos que no. Lo único que hacíamos era convertir los filtros de Excel, en lecturas profundas para detectar las constantes y variables culturales que a priori habíamos construido y convertido en categorías de análisis, además de utilizar marcadores para facilitar la búsqueda en las matrices que habían sido diseñadas. Después de todo, el tipo de matriz de observación utilizada era sobre observables muy concretas que facilitaban el trabajo de procesamiento de información. Reconocíamos que muchos datos importantes se iban a quedar fuera, pero que no movía otra cosa que no fuera cumplir con los tiempos. Además, la cantidad de "carnita" con la que contaba y el conecimiento pleno de la información que teníamos, no podía reducirse a ese acto de "magia académica" llamada "sacada de la manga", y que además lo compartíamos.

Lo que a continuación realizamos fue afinar el diseño de las matrices donde concentramos toda la información, tomando como punto de partida las dimensiones primarias que en toda situación social se presentan según la propuesta de Spradley: los sujetos, las acciones, los espacios y los objetos. El corpus de información obtenido en campo, sería procesado considerando estos momentos; cosa que se hacía relativamente sencillo pues la matriz de registro empleada a lo largo de la investigación, dividía la información de acuerdo a estas variables; además de contar con otra ficha que trabajaba 
directamente sobre eventos que "apuntaban" a las categorias de análisis de nuestro modelo heurístico..

Por otro lado, tendría que establecer una articulación empírico-conceptual con las categorías trabajadas en el cuerpo teórico. Realmente este proceso no fue fácil. El trabajo fuerte consistió en el establecimiento de una "red semántico cultural", que para nosotros hacía objetiva la información empírica, misma que formaban parte de las categorías teóricas con las había estado trabajando; además de permitir la sistematización de la información recabada. Fueron cuatro matrices las principales, a partir de las cuales fueron emergiendo los datos claves que permitían realizar una serie de inferencias fundamentadas en la perspectiva teórica propuesta. Posteriormente, con apoyo de nuestra asesora, se elaboró una ficha de análisis comparativo continuo, y con ella, un mejor trabajo sintético de información que proporcionaba evidencia empírica sobre la que se podía hacer un análisis más fino. Es importante reconocer que la inclusión de otras categorías por ella sugeridas, junto a la esquematización teórica que nos presentó y donde hallamos la articulación final de lo que deberíamos hacer, contribuyó enormemente en las maneras de reconfiguración para la parte conclusiva de la investigación.

A continuación, buscando que lo dicho y escrito quedara lo más explícito posible, diseñamos cuadros, fichas de análisis, una serie de mapas conceptuales, ${ }^{15}$ redes categoriales que permitieran mostrar lo obtenido en campo y cruzarlo con la teoría, para con ello, tener mejor sustento en nuestras argumentaciones, y así mostrar que el puñado de intuiciones de inicio, eran superadas o se veían fortalecidas por lo que estaba siendo evidenciado a través de esa información ya procesada, sistematizada y confrontada con la teoría. Lo que restaba, entonces, era la interpretación.

A partir de esto, lo que también preocupaba, era cómo trasladar todo esto a discurso, a textualidad. Respuesta que no encontramos hasta el momento en que hubo la consulta de Clifort Geertz (1994), quien reconoce un nivel de análisis de los fenómenos socioculturales, en todos aquellos productos textuales de un investigador, cercanos a la ficción si se consideran que aun con lo riguroso, es un acto de mediación cultural sobre fenómenos ya interpretados por los mismos actores sociales.

Cabe señalar que adoptamos como marco metodológico la llamada hermenéutica profunda a la que hace referencia John B. Thompson (1998), para el estudio de la dimensión simbólica de los fenómenos culturales en la constitución significativa de las acciones sociales. Desde allí y con una mayor claridad para esta parte del trabajo, la decisión fue proceder a escribir.

15. Para explicitar y darle sustento mejor a los esquemas analíticos y los gráficos, nos recomendó estudiar a Edward De Bono y su lógica fluida, quien daría mayor soporte a ese sistema de análisis propuesto. 
Sin embargo, la "imperante" necesidad de hacer objetivo lo empírico junto a lo teórico, llevó a sentarnos a teclear, teniendo en claro otra preocupación: qué lenguaje utilizar, después de todo la academia "reconoce" un tipo de discurso, la ciencia otro (como ya quedó esbozado en el apartado anterior) y nuestra orientación y gusto literario venía "chocando" con esto. Fue al tropezarnos con textos de Renato Ortiz (1999), Jorge González (1994) y Oliver Sack (1995), cuando comprendimos era posible elaborar un tipo de discurso que fuera más allá del "acartonamiento" y la ortodoxia propuestos por la academia clásica. Ante la certeza de entender, como decía el primer autor, que la legitimación de lo objetivo de un trabajo científico va más allá de las buenas maneras en el hablar académico (el tan discutido descentramiento del discurso personalizado para evitar todo rasgo de subjetividad, sobre el que es hora de comenzar a dudar), la versión final del "borrador de tesis" indagó en las posibilidades del lenguaje, con su riqueza, matices y "rigor" reflexivo. En el caso de González, habría que reconocer las formas de nombrar las cosas y plantear sus argumentos, para reconocer en sus formas lingüísticas lo que en algún momento dijera: habría que leer poesía, ver cine y acercarse a literatura, para hacer más rica la experiencia de escribir la ciencia. Mientras que en el caso de Sack, es la muestra de alguien venido de las llamadas ciencias "duras", capaz de establecer un discurso más cercano a lo literario que a lo científico.

En ese lapso, hubo un gusto por lo que estaba haciéndose. Por segunda vez se tenía la certeza que lo hecho podía acercarse a un trabajo de tesis y no a una obra ensayística. Mapas, cuadros sinópticos, esquemas (fundamentados en la lógica de flujo y los bucles propuestos por De Bono, ahora convertidos ámbitos comunicativos, para "validarlos" teóricamente), fueron incorporados al cuerpo del texto, buscando fundamentar nuestras argumentaciones. Junto a estas reflexiones, el recurso de las citas de las entrevistas in situ, ${ }^{16}$ que fueran forjando y dando matices a lo que se iba escribiendo.

Por otro lado, no podemos dejar de lado el hecho de desconocer, académicamente, cómo se debería emplear la información contenida en el diario de campo. De tal forma, fuimos integrando nuestro engranaje discursivo con los "deslices" reflexivos provocados en campo: dudas, certezas, preguntas, siempre buscando evitar términos que fueran más allá de los compromisos que en la parte metodológica habíamos acordado. Ya describíamos, ya analizábamos,

16. Esta posibilidad de recuperación del discurso endocultural, proporcionó una condición importante en el trabajo de análisis, pues nos orientó para la creación de los dominios culturales, el análisis comparativo constante y para incorporar sus voces en el momento de convertir la interpretación en textos. La experiencia en todo sentido, fue enriquecedora, pues en su momento reprodujo formas discursivas en las que nos encontramos inmersos y de la cual salimos ayudados por el trabajo reflexivo que toda relación sujeto-objetosujeto, requicre. 
pero sobre todo buscábamos interpretar desde la hermenéutica, para comprender esas formas simbólicas de la cultura que nuestro objeto de estudio revelaba.

Finalmente, reconocemos que pudo haber una falta de solvencia metodológica al inicio, pero pensamos que el trabajo realizado después, estableció un rigor, pero sobre todo, trató de ser honesto a partir del reconocimiento de las mismas carencias que nos acompañaron en este recorrido iniciático. Seguro las cosas daban para más, tal y como las planteamos de entrada; sin embargo, por razones ya expuestas y algunas más que pudiéramos nombrar, quizá no se pudo llegar a entrar en la dimensión analítica que se pretendía con esta investigación en sus inicios; pero después de todo, como alguien dijo: cuando se mete en este tipo de trabajo, al final muchas cosas cambian y otras tantas se dejan de lado. Y de todo ello, lo más importante es que esto no desmerece lo ahora entregado.

Hoy que pergeñamos estas líneas, lo que "rebota" en la cabeza es si lo dicho aquí, vale la pena. No sabemos si esto reste en mucho al trabajo, pero creemos que si se da la oportunidad de hacer un trabajo crítico y por momentos reflexivo, lo que menos esperamos para con uno (que no tanto para los demás), es un momento de honestidad.

Quedan pues aquí estas palabras a suerte de anecdotario de viaje. Tantas cosas como pudimos se han dicho, otras se han ido perdiendo a lo largo de esta travesía que, en la narración de los hechos y las cosas olvidadas, seguro quedaron guardadas para alguna otra ocasión.

\section{Referencias}

González, Jorge. (1994) Mas (+) cultura(s). Ensayos sobre realidades plurales. CNCA colec. Pensar la cultura, 1a. edición, México.

Geertz, Cliffort. (1994) La interpretación de las culturas, editorial Gedisa, España.

Geertz, Cliffort. Conocimiento local. Ensayo sobre la interpretación de las culturas, Ed. Paidós, México.

De Bono, Edward. (1996) Lógica fluida. La alternativa a la lógica tradicional, editorial Paidós, México.

Ortiz, Renato. (1999) “Ciencias sociales, globalización y paradigmas" en Pensar las ciencias sociales hoy. Reflexiones desde la cultura, Reguillo Cruz, Rossana y Raúl Fuentes Navarro (coords.), ITESO, México.

Poblete, Sergio. "La descripción etnográfica. De la representación a la ficción” en Cinta de moheció, núm. 6, septiembre de 1999. Facultad de 
78 De una necesidad a posteriori

Ciencias Sociales, Universidad de Chile. http://rehue.csociales.uchile.cl/publicaciones/moebio/o6/frames 05.htm.

Sack, Oliver. (1995) El hombre que confundió a su mujer con un sombrero, Grijalbo, México.

Spradley, James. (1980) Participant observation, Holt, Rinehart and Winston, New York, USA.

Thompson, John B. (1996) Ideología y cultura moderna, UAM-Xochimilco, $2^{\mathrm{a}}$. Edición, México. 